\title{
Virtual hysteroscopy: a new non invasive approach for the assessment of uterine cavity
}

\author{
Paulo Sérgio Cossi ${ }^{1}$, Heron Werner ${ }^{2}$, Wellington P. Martins ${ }^{3}$, Alberto Borges Peixoto ${ }^{1,4}$, \\ Edward Araujo Júnior ${ }^{1}$
}

${ }^{1}$ Department of Obstetrics, Paulista School of Medicine, Federal University of São Paulo, São Paulo-SP, ${ }^{2}$ Clínica de Diagnóstico por Imagem, Rio de Janeiro-RJ, ${ }^{3}$ Department of Obstetrics and Gynecology, Ribeirão Preto Medical School, University of São Paulo (FMRP-USP), Ribeirão Preto-SP, ${ }^{4}$ Mario Palmério University Hospital - University of Uberaba, Uberaba-MG, Brazil.

\begin{abstract}
Endometrial pathology is frequent in premenopausal women and the diagnostic hysteroscopy is considered the gold standard for assessing endometrial cavity and congenital uterine anomalies. However it is an invasive and uncomfortable procedure. Saline contrast hysterosalgingography and hysterosalpingo-contrast sonography allow detailed assessment of the uterine cavity. We propose a virtual hysteroscopy from three-dimensional saline and three-dimensional hysterosalpingo-contrast sonography scan data to generate a virtual reality as a new noninvasive procedure for assessing the uterine cavity in uterine congenital anomaly and endometrial pathology cases as well

Keywords: virtual hysteroscopy; three-dimensional hysterosalpingo-contrast sonography; three-dimensional saline hysterosalgingography; septate uterus; endometrial polypus.
\end{abstract}

Endometrial pathology occurs in $40 \%$ of premenopausal women, with abnormal bleeding, uterine fibroids $(30 \%)$, and polyps $(30 \%)$ being the most common findings [1]. The assessment of endometrial cavity can be performed by ultrasonography, contrasted hysterosalgingography (HSG), and diagnostic hysteroscopy (HCT) [2]. Diagnostic hysteroscopy is considered the gold standard for assessing endometrial pathologies and congenital uterine anomalies [3]; however, it is an invasive and uncomfortable procedure [4]. Compared with diagnostic hysteroscopy, saline contrast HSG is a noninvasive procedure with similar accuracy in the diagnosis of focal endometrial pathology [5]. Three-dimensional (3D) hysterosalpingo-contrast sonography (HyCoSy) that associ-

Received 29.11.2016 Accepted 23.01.2017

Med Ultrason

2017, Vol. 19, No 2, 216-217

Corresponding author: Prof. Edward ARAUJO JÚNIOR, PhD

Rua Belchior de Azevedo, 156 apto. 111

Torre Vitoria, São Paulo-SP, Brazil

CEP 05089-030

Phone/Fax: +55-11-37965944;

Email: araujojred@terra.com.br ates second-generation agents, such as SonoVue, with new software (Coded contrast imaging; GE Healthcare, Zipf, Austria) allows detailed assessment of the uterine cavity and fallopian tube patency [6].

We propose a virtual hysteroscopy from 3D saline and $3 \mathrm{D}$ HyCoSy scan data to generate a virtual reality as a new noninvasive procedure for assessing the uterine cavity in uterine congenital anomaly and endometrial pathology cases as well as the fallopian tube patency.

A 25-year-old woman with pain in the lower abdomen-type colic that increased in intensity in the last menstrual period was examined. Her menarche was at 14 years of age, and with regular menstrual periods. She had to two previous pregnancies with one term labor and one miscarriage without curettage. A 3D HyCoSy was performed with a Voluson E6 apparatus (GE, Healthcare, Milwaukee, WI, USA) equipped with an endovaginal probe (RIC 5-9D). Initially, a specific catheter for HSG (5-Fr) with a silicone balloon $(1.5 \mathrm{~mL})$ (Cook Medical, Spencer, IN, USA) was introduced into the cervical canal and inflated with 3-mL distilled water. In sequence, the protocol-coded contrast imaging mode was activated, 


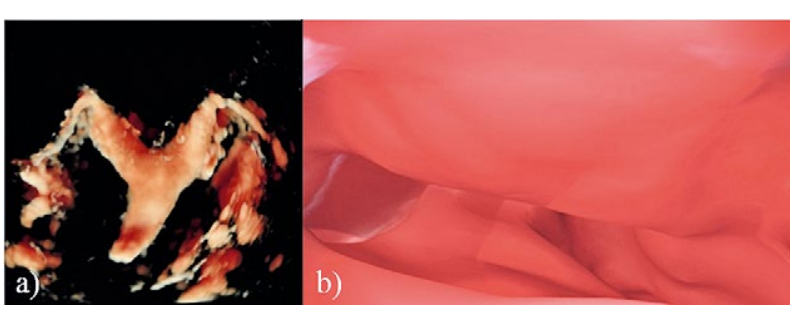

Fig 1. a) Three-dimensional (3D) hysterosalpingo-contrast sonography (HyCoSy) demonstrating a septate uterus; b) 3D virtual hysteroscopy in the septate uterus.

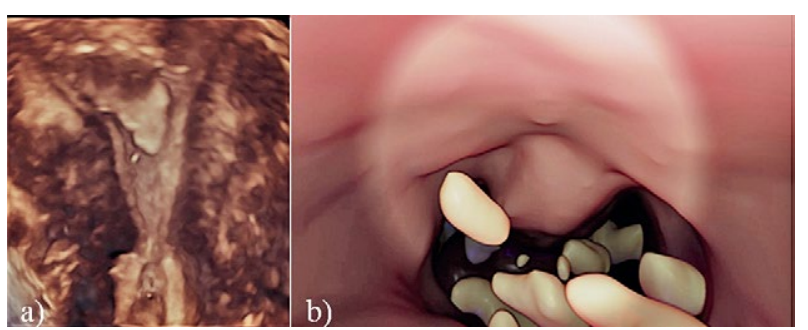

Fig 2. a) Three-dimensional (3D) saline contrast hysterosalgingography (HSG) demonstrating a large endometrial polypus in the fundus; b) 3D virtual hysteroscopy in the uterus demonstrating several endometrial polyps.

and the pelvis became totally anechoic. By introducing contrast, the uterine cavity and tubal patency were delineated using hyperechoic fluid, and finally, the contrast overflowed to the abdominal cavity. A 3D HyCoSy confirmed the diagnosis of septate uterus. A 3D model of the uterus was created by overlapping image layers from the 3D volume datasets using the software Mimics (Materialize, Leuven, Belgium). Contrast detection was applied to areas of interest to delineate the surface of the uterine cavity; the resulting 3D virtual model was exported using the standard triangular language file format and then converted into an OBJ file using the 3D modeling software MAYA (Autodesk, San Rafael, CA, USA). The latter program allowed virtual positioning of the observation cameras while working with multiple on-screen windows, and lighting parameters were adjusted to optimize visualization. Lastly, a path was plotted through the 3D model to create a simulated movie for analyzing the uterine cavity by 3D virtual hysteroscopy (fig 1, Supplementary material on journal website- Video S1).

A 39-year-old woman who had vaginal bleeding for 2 days after the menstrual period, with pain in the lower abdomen, mainly in the right iliac fossa, was examined. Her menarche was at 12 years of age, with regular menstrual periods and dysmenorrhea. She was nulligest. A 3D saline contrast HSG revealed a submucous myoma and a large endometrial polypus in the fundus as well as other small endometrial polyps in the entire endometrial cavity. A 3D virtual hysteroscopy, as demonstrated above, clearly demonstrated the endometrial polypus (fig 2, Supplementary material on journal website- Video S2).

A 3D virtual navigation to assess the bronchia of fetuses with cervical teratomas was described previously by our group in obstetrics [7]; however, to the best of our knowledge, this is the first description in gynecology literature on the use of 3D virtual navigation for the diagnosis of congenital uterine anomaly and endometrial pathology as well as fallopian tube patency. We believe that $3 \mathrm{D}$ virtual hysteroscopy can provide noninvasive complementary diagnostic information regarding congenital uterine anomaly or endometrial pathology. New studies are necessary to evaluate whether such information is relevant to improve diagnostic test accuracy, patient understanding, or better surgery planning.

\section{Conflict of interest: none}

\section{References}

1. Emanuel MH, Verdel MJ, Wamsteker K, Lammes FB. A prospective comparison of transvaginal ultrasonography and diagnostic hysteroscopy in the evaluation of patients with abnormal uterine bleeding: clinical implications. Am J Obstet Gynecol 1995;172:547-552.

2. Gronlund L, Hertz J, Helm P, Colov NP. Transvaginal sonohysterography and hysteroscopy in the evaluation of female infertility, habitual abortion or metrorrhagia. A comparative study. Acta Obstet Gynecol Scand 1999;78:415-418.

3. Makled AK, Farghali MM, Shenouda DS. Role of hysteroscopy and endometrial biopsy in women with unexplained infertility. Arch Gynecol Obstet 2014;289:187-192.

4. Berger A, Batzer F, Lev-Toaff A, Berry-Roberts C. Diagnostic imaging modalities for Müllerian anomalies: the case for a new gold standard. J Minim Invasive Gynecol 2014;21:335-345.

5. Milingos S, Kallipolitis G, Stefanidis K, Sklia J, Makris N, Loutradis D, Antsaklis A, Michalas S. Saline contrast hysterosonography in infertile patients and in women with abnormal uterine bleeding. Eur J Gynaecol Oncol 2005;26:564-567.

6. Exacoustos C, Di Giovanni A, Szabolcs B, Binder-Reisinger H, Gabardi C, Arduini D. Automated sonographic tubal patency evaluation with three-dimensional coded contrast imaging (CCI) during hysterosalpingo-contrast sonography (HyCoSy). Ultrasound Obstet Gynecol 2009;34:609-612.

7. Werner H, Dos Santos JR, Fontes R, Daltro P, Gasparetto E, Marchiori E, Campbell S. Virtual bronchoscopy in the fetus. Ultrasound Obstet Gynecol 2011;37:113-115. 